\title{
A lower-limit flux for the extragalactic background light
}

\author{
T. M. Kneiske ${ }^{1}$ and H. Dole ${ }^{2}$
}

\author{
1 Institut für Experimentalphysik, University of Hamburg, Luruper Chaussee 149, 22761 Hamburg, Germany \\ e-mail: tanja.kneiske@desy.de \\ 2 Institut d'Astrophysique Spatiale, Université Paris Sud 11 \& CNRS (UMR 8617), Bât 121, 91405 Orsay, France \\ Received 6 March 2009 / Accepted 14 January 2010
}

\section{ABSTRACT}

\begin{abstract}
Context. The extragalactic background light (EBL) contains information about the evolution of galaxies from very early times up to the present. The spectral energy distribution is not known accurately, especially in the near- and mid-infrared range. Upper limits and absolute measurements come from direct observations which might be be polluted by foreground emission, while indirect upper limits can also be set by observations of high energy gamma-ray sources. Galaxy number counts integrations of observable galaxies, missing possible faint sources, give strict lower limits.

Aims. A model is constructed, which reproduces the EBL lower limit flux. This model can be used for a guaranteed minimum correction of observed spectra of extragalactic gamma-ray sources for extragalactic absorption.

Methods. A forward evolution model for the metagalactic radiation field is used to fit recent observations of satelites like Spitzer, ISO, Hubble and GALEX. The model is applied to calculate the Fazio-Stecker relation, and to compute the absorption factor at different redshifts and corrected blazar spectra.

Results. A strict lower-limit flux for the evolving extragalactic background light (and in particular the cosmic infrared background) has been calculated up to a redshift of five. The computed flux is below the existing upper limits from direct observations, and agrees with all existing limits derived from very-high energy gamma-ray observations. The corrected spectra still agree with simple theoretical predictions. The derived strict lower-limit EBL flux is very close to the upper limits from gamma-ray observations. This is true for the present day EBL, but also for the diffuse flux at higher redshift.

Conclusions. If future detections of high redshift gamma-ray sources require a lower EBL flux than derived here, the physics assumptions used to derive the upper limits have to be revised. The lower-limit EBL model is not only needed for absorption features in active galactic nuclei and other gamma-ray sources, but is also essential when alternative particle processes are tested, which could prevent the high energy gamma-rays from being absorbed. It can also be used for a guaranteed interaction of cosmic-ray particles. The model is available online.
\end{abstract}

Key words. diffuse radiation - Galaxy: formation - infrared: galaxies

\section{Introduction}

Diffuse extragalactic background radiation has been observed over a broad range of the energy spectrum from radio to high energy gamma-rays. A main contribution at almost all wavelengths (except for the cosmic microwave background, CMB) are faint point sources (sometimes unresolved), emitting in the energy band of interest. Therefore the extragalactic background radiation turns out to be a good tool to study global parameters of source populations and universal physics. The optical to infrared extragalactic diffuse radiation, also called extragalactic background light (EBL), is the relic emission of galaxy formation and evolution and is produced by direct star light (UV and visible ranges) and light reprocessed by the interstellar dust (infrared to sub-millimeter ranges). Minor contribution may include genuine diffuse emission (e.g. galaxy clusters described in Chelouche et al. 2007) or other faint sources (e.g. population III stars as shown in Raue et al. 2009).

While it is possible to measure extragalactic diffuse emission in the sub-mm range (Puget et al. 1996; Hauser et al. 1998; Hauser \& Dwek 2001), the EBL is difficult to measure directly in the infrared because of strong foreground contamination. Thus upper limits have been derived by observing the isotropic emission component (see Hauser \& Dwek 2001; Kashlinsky 2005 for reviews, as well as Lagache et al. 2005; Dole et al. 2006). Lower limits can be derived by integrated galaxy number counts.
The method has been improved during the last years by sensitive telescopes like Spitzer (Werner et al. 2004). This technique gives good constraints at wavelengths shorter than 24 microns. At larger wavelengths, higher confusion and lower sensitivities lead to very small lower limits. To overcome the poor constraints at far-infrared wavelengths, a stacking analysis of near- and midinfrared sources is used (e.g. Dole et al. 2006) to significantly resolve the cosmic infrared background (CIB), leading to constraining lower limits.

Other constraints on the EBL are coming from the study of distant sources of very high energy gamma-ray emission. High-energy gamma rays traveling through intergalactic space can produce electron-positron pairs in collisions with low energy photons from the extragalactic background light (Nikishov 1962; Goldreich \& Morrison 1964; Gould \& Schreder 1996; Jelley 1966). Despite this effect, Cherenkov telescopes have discovered a great number of extragalactic high energy gammaray sources at unexpected high redshifts. The discovery of 3C279 by the MAGIC telescope collaboration (Albert et al. 2008) shows that $80-500 \mathrm{GeV}$ gamma-rays photons can travel distances from redshift $z=0.536$ without being too heavily absorbed. From the observation of H2356-309 and 1ES 1101232, the HESS collaboration derived an upper limit for the EBL between 1 and 4 micron (Aharonian et al. 2006), which is very close to the optical number counts by the Hubble Space Telescope (Madau \& Pozzetti 2000). They verified their result in 
Aharonian et al. (2007a) with the BL Lac 1ES 0347-121 and extended their limit to the mid-infrared using 1ES $0229+200$ Aharonian et al. (2007b). The caveat is that the upper limit strongly depends on the assumption of the intrinsic blazar spectrum.

Different types of models for the EBL flux have been developed. The simplest method (backwards evolution) extrapolates present day data or template spectra to high redshift in a certain wavelength range (for the most recent ones see Chary \& Elbaz 2001; Malkan \& Stecker 2001; Totani \& Takeuchi 2002; Lagache et al. 2003, 2004; Xu et al. 2003; King et al. 2003; Stecker et al. 2006; Franceschini et al. 2008). Cosmic chemical evolution models self-consistently describe the temporal history of globally averaged properties of the Universe (Pei et al. 1999), but fall short when it comes to comparisons with data of individual galaxies. Semi-analytical models are invoking specific hierarchical structure formation scenarios to predict the metagalactic radiation field (MRF, i.e. the EBL at various redshifts) (e.g. Balland et al. 2003; Primack 2005). The model used in this paper is an updated version of the Kneiske et al. (2002, 2004) forward evolution model. Simple stellar population models are used to describe the evolution of stars in the universe from their very first formation up to the present. Not only the physics of stars but also the composition and spatial distribution of the interstellar medium are taken into account.

In this work lower-limit EBL data are used to derive a lowerlimit EBL flux model. In the next section, the data and their uncertainties are discussed. The minimum EBL flux model is derived in the third section by choosing parameters for the global star formation and the interstellar medium. The results are presented in the fourth section, together with the resulting optical depth for gamma-rays in the universe. Throughout this paper, a cosmology with $h=0.72, \Omega_{\mathrm{M}}=0.3$ and $\Omega_{\Lambda}=0.7$ is adopted.

\section{Current lower limits on the cosmic optical and infrared backgrounds}

Lower limits on the extragalactic background light measurements are reviewed briefly. Most are derived from the integration of number counts, not from direct measurements of surface brightness, which is subject to strong foreground emission contamination. This method is based on the simple counting of detected galaxies on a given sky area of a deep survey, a completeness correction, and the flux integration of the number counts. Variance due to large-scale structure may affect the results and is usually taken into account in the error bars. However, another source of uncertainty at near-infrared wavelengths is the usually poor detected galaxy statistics at high flux densities and the subtraction of stars; these uncertainties affect the number counts at high flux densities and can give different results when integrating them to get the background lower limit. Any model of the EBL should thus lie above these observed limits. In the past not all EBL models met this criterion and are therefore not realistic and in contradiction with the data. The lower limit data are shown in Fig. 3 as data points with the errors discussed below.

\subsection{Ultraviolet and visible EBL}

Counts and integration were done by $\mathrm{Xu}$ et al. (2005) (GALEX); Brown et al. (2000) and Gardner et al. (2000) (HST/STIS); Madau \& Pozzetti (2000) and Totani et al. (2001) (HST/WFPC2).

\subsection{Near- and mid-infrared EBL}

The integration of number counts on deep surveys done with the HST was done by Madau \& Pozzetti (2000) and Thompson 2003 Thompson et al. (2007) (NICMOS), and Totani et al. (2001) (SUBARU).

Fazio et al. (2004a) obtained number counts with Spitzer/IRAC at 3.6, 4.5, 5.8 and $8.0 \mu \mathrm{m}$, and derived lower limits. These counts have been confirmed by Magdis et al. (2008) at these four wavelengths and by Franceschini et al. (2006) at $3.6 \mu \mathrm{m}$. At $8.0 \mu \mathrm{m}$, however, Franceschini et al. (2008) recomputed the counts at larger flux densities with better statistics and re-integrated the whole number counts; they claim that their integration gives a 50\% smaller value that Fazio et al. (2004a). The value published by Franceschini et al. (2008) will be used as a lower value at $8.0 \mu \mathrm{m}$. Similarly the $5.8 \mu \mathrm{m}$ estimate would need to be recomputed. At $3.6 \mu \mathrm{m}$, Levenson \& Wright (2008) integrated the extrapolated number counts (with constraints from the image noise) and came close to the DIRBE minus 2MASS value, giving an estimate of the CIB at this wavelength. As a strict $3.6 \mu \mathrm{m}$ lower limit, the Fazio et al. (2004a) value is used. It should be noticed though that IRAC counts at this wavelength may not be that reliable when integrated to give CIB lower limits, although number counts are very accurately measured in deep surveys at faint flux densities (e.g. agreement between Fazio et al. (2004a), Franceschini et al. (2006) and Magdis et al. $(2008)$ at $3.6 \mu \mathrm{m})$. Counts are contaminated by the presence of bright and faint stars and extended local galaxies, biasing the measure at high and intermediate flux densities, where deep surveys have very poor statistics. Deep and shallow surveys have better statistics, but the star contribution subtraction could be inaccurate and could dominate the systematics uncertainty. Nevertheless the data point will be included in our analysis, where the error bars represent the large uncertainties.

In the mid-infrared, the counts by Elbaz et al. (2002) at $15 \mu \mathrm{m}$ with ISOCAM are used. At $24 \mu \mathrm{m}$ with Spitzer/MIPS, the counts by Papovich et al. (2004), Marleau et al. (2004) and Chary et al. (2004), Rodighiero et al. (2006) are used. At these wavelengths, contributions of stars and extended galaxies are negligible. The stellar spectra can be described Rayleigh-Jeans approximation and the point spread functions are larger than 6 arcsec. The lower limits are therefore reliable.

\subsection{Far-infrared and sub-millimeter EBL}

Above $30 \mu \mathrm{m}$ wavelength, another method than integrating the number counts is used, because individual detected far-infrared sources do not contribute more than $25 \%$ to the background (e.g. Dole et al. (2004), Frayer et al. (2006) except in the GOODS $70 \mu \mathrm{m}$ survey (about 60\% Frayer et al. (2006b). This method consists of stacking a longer-wavelength signal at the position of known short wavelength sources and then measuring the resulting total flux, which is also a lower limit. At $70 \mu \mathrm{m}$ and $160 \mu \mathrm{m}$, the lower limits of Dole et al. (2006) obtained with a stacking analysis of Spitzer/MIPS $24 \mu \mathrm{m}$ sources is used. The submillimeter $C O B E / F I R A S$ spectrum of direct detection comes from Lagache et al. (2000).

\section{Lower-limit EBL model}

In this section an EBL model is constructed which reproduces the EBL flux lower limits from source counts. The EBL model is described in detail in Kneiske et al. (2002) and the main features are summarized below. The idea is to describe cosmological 
T. M. Kneiske and H. Dole: A lower-limit flux for the extragalactic background light

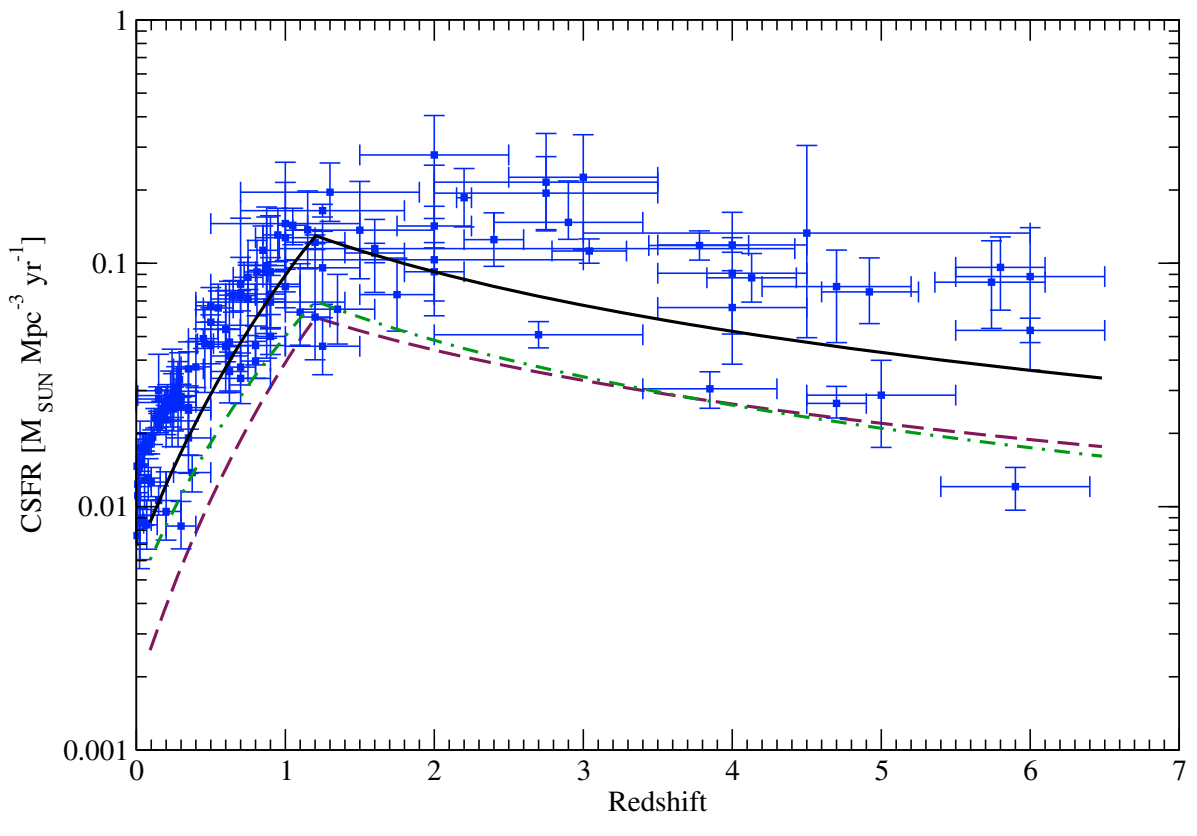

Fig. 1. Comoving cosmic star formation rate. The data are taken from 2006. The solid line shows the model total star formation rate, while the dashed and dashed-dotted line accounts the contribution from dust-poor and dust-rich regions respectively.

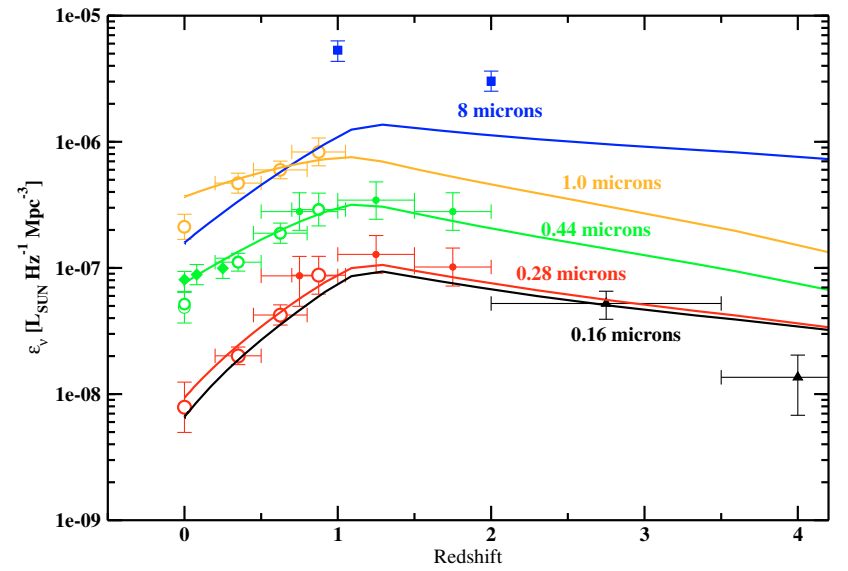

Fig. 2. Comoving emissivity as a function of redshift. The lines are calculated for the wavelength indicated in the figure and have to be compared with the data points of the same color. Data come from: Ellis et al. (1996), Lilly et al. (1996), Connolly et al. (1997), Pozzetti et al. (1998), Caputi et al. (2007).

stellar evolution with a simple stellar population model depending on different stellar masses. The cosmological evolution is set by an input comoving star formation rate density (SFR). The model computes emissivities and the EBL flux, which can be directly compared with observations at individual wavelengths. Two different star forming regions are distinguished phenomenologically: "optical" star forming regions with low extinction due to the presence of dust $(E(B-V)=0.06)$, and "infrared" star forming regions with higher extinction aiming at reproducing the emission properties of luminous and ultraluminous infrared galaxies (LIRG and ULIRG; $E(B-V)=0.8$ ). For these two populations, spectral energy distributions (SED) are generated with a spectral synthesis model, adding a consistent model accounting for dust absorption and reemission. Three components of dust are taken into account by modified black body spectra with different temperatures. The goal is thus to fit the EBL observed lower limit by adjusting the input SFR and dust parameters.

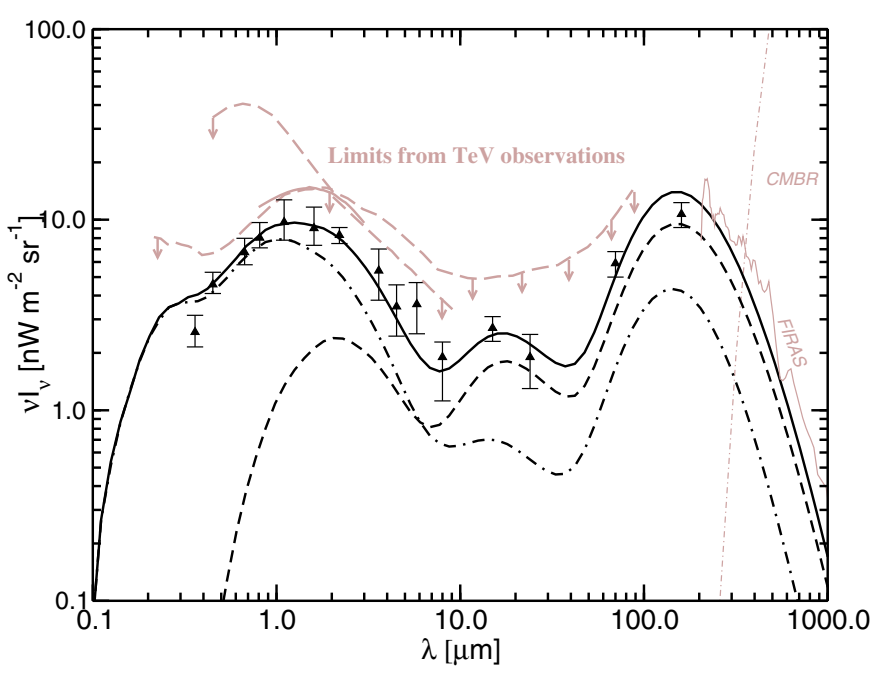

Fig. 3. Extragalactic Background Light Spectral Energy Distribution. Data are lower limits (filled triangles), discussed in Sect. 2. The total model flux is shown as black solid line, together with the contribution from dust rich (dashed line) and dust poor star forming regions (dotdashed line). The red dashed line are model-dependant upper limits on the EBL as derived from high energy blazar observations (Aharonian et al. 2006; Aharonian et al. 2007a,b; Albert et al. 2008; Mazin \& Raue 2007). Other long-wavelength detections are plotted: the submillimeter EBL and the CMB.

The EBL model flux was fitted to the observed lower limits summarized in the last section by integrating the emissivities on the redshift range zero to two. This takes into account the fact that data are only able to resolve galaxies up to a certain redshift, which depends on the flux limit of the instrument and the survey. It is not possible to give the exact maximum redshift for each survey, since the redshift is not known automatically for each detected source. The chosen maximum redshift of two seems a good average for most surveys taken into account. Our result is only weakly dependent on this parameter. The model parameters were chosen to minimize the $\chi^{2}$ between EBL observed limits and the model. 


\section{Results and discussion}

\subsection{Cosmic star formation rate and emissivity}

The model output cosmic star formation rate is shown in Fig. 1. It is lower by a factor of two to three than the data compiled by Hopkins \& Beacom (2006). This is not surprising, as a lower limit EBL is used, which by definition is missing some amount of emission yet the shape is consistent with the data.

Since the star formation rate is a model-dependent value which shows a wide range of scatter, it is useful to compare the model emissivities at different redshifts with integrated luminosity functions at various wavelengths. As shown in Fig. 2, the agreement between optical $(\lambda \leq 1 \mu \mathrm{m})$ data and the model emissivity is good for redshifts below three. The model, however, is underestimating the emissivity at $8 \mu \mathrm{m}$ by a factor three to five. The origin of this discrepancy might be twofold: 1) the simplistic galaxies' spectral energy distribution used, which lack detailed aromatic bands and have a very small grains continuum description; and 2) a slight overestimation of the observed $8 \mu \mathrm{m}$ emissivity, obtained trough the rest-frame $8 \mu \mathrm{m}$ luminosity function integration (Caputi et al. 2007) and an extrapolation to the infrared bolometric luminosity density. Despite the care taken, this last operation might slightly overestimate the emissivity. This might be the reason why the model does not strongly disagree with the EBL shape at $8 \mu \mathrm{m}$ (Fig. 3), despite a disagreement with the $8 \mu \mathrm{m}$ emissivity.

\subsection{Extragalactic background light (EBL)}

The observed EBL lower limits (Sect. 2) are plotted in Fig. 3 together with the model. The model reproduces the data well, keeping in mind that a physical model was used instead of a functional fit, and that the minimum $\chi^{2}$ used. Almost all EBL flux (wavelengths $0.3 \leq \lambda \leq 160 \mu \mathrm{m}$ ) comes from galaxies up to a redshift of two, as expected (e.g. Lagache et al. 2005). There is no significant change in the computed EBL spectrum when including emission from redshifts above two, since the cosmic star formation rate drops by half an order of magnitude. The robustness of our EBL derivation is checked by integrating the emissivities up to a redshift of $z=5$ : this does not change the final result by more than $4 \%$. The optical and infrared EBL are dominated by their respective components (optical and infrared galaxies), and the transition region between both contributions, located around 5 microns, can be probed by Spitzer. The 5.8 micron data point lies above our model flux by more than $1 \sigma$. As discussed in Sect. 2, this point might suffer from a poor statistics. At 8 micron, the new estimate of Franceschini et al. (2008) lies on our model, but the Fazio et al. (2004a,b) estimate is higher. While a consistent new estimate of all IRAC points would be needed, it is possible yet to conclude if this discrepancy is a common feature of EBL models (see also Franceschini 2008; Primack et al. 2008), and/or if the data points around $5 \mathrm{mi}-$ crons are overestimated (this last possibility cannot be ruled out, as discussed in Sect. 2). Finally, our EBL model lies below the observed upper limits derived from gamma-ray observations, as expected.

\subsection{EBL and $\gamma$-ray absorption at high redshift}

The lower limit EBL model can be used to calculate the optical depth for photon-photon pair production. The effect is mainly important for extragalactic sources like blazars (Salamon \& Stecker 1998; Primack et al. 1999; Kneiske et al. 2004) or
Table 1. Model input parameters (definitions see Kneiske et al. 2004).

\begin{tabular}{lllll}
\hline \hline & $\alpha$ & $\beta$ & $z_{p}$ & $\begin{array}{l}\dot{\rho}_{*}\left(z_{p}\right) \\
{\left[M_{\odot} \mathrm{Mpc}^{-3} \mathrm{yr}^{-1}\right]}\end{array}$ \\
\hline Strict lower-limit model & & & & \\
$S F R_{O P T}$ & 3.5 & -1.2 & 1.2 & 0.07 \\
$S F R_{L I G}$ & 4.5 & -1.0 & 1.2 & 0.06 \\
$f_{\text {esc }}=0$ & & & & \\
$c_{2}=10^{-24}$ & & & & \\
\hline
\end{tabular}

gamma-ray bursts. The absorption can result in a drastic change of the high energy spectrum or even make it impossible to observe the source at all at gamma-ray energies. The effect of absorption for extragalactic gamma-ray sources at different redshift is shown in Fig. 4. The EBL flux is plotted next to the absorption factor $\exp (-\tau)$ at the same redshift. The spectral region of the EBL flux responsible for the so cut-off region is indicated by vertical red lines and arrows. The cosmic microwave background is also plotted as a dot-dashed line on the right of the EBL flux diagram. The results of our new lower-limit EBL model are compared with the so called "best-fit" EBL model from Kneiske et al. (2004). It is clearly visible that a lower EBL flux leads to an absorption closer to one, which means less absorption of gammaray photons in the cut-off region.

\subsection{Fazio-Stecker relation}

The attenuation of gamma-rays can also be expressed by the Fazio-Stecker relation, also known as the gamma-ray horizon. It is shown in Fig. 5 for a source-independent description. The redshift of a high energy gamma-ray source is plotted against gamma-ray energy for an optical depth $\tau_{\gamma \gamma}\left(E_{c}, z\right)=1$ (black line), $\tau_{\gamma \gamma}\left(E_{c}, z\right)=2$ (green line), $\tau_{\gamma \gamma}\left(E_{c}, z\right)=3$ (red line). These lines are calculated by the lower-limit model derived in this work. Limits from blazar observations are plotted as well taken from Albert et al. (2008). The blazars all lie in the transparent region $(\tau<1)$ according to our model. For a given energy, blazars at a slightly higher redshift than already measured might be detected. All data agree with the lower limit model. Although a lower-limit EBL has been used, there is a little room left for a higher EBL flux resulting in a higher optical depth for high energy gamma-rays.

Finally the result is compared with the models by Primack (2005), Albert et al. (2008) and Stecker et al. (2006) (dashed, dot-dot-dashed, and dot-dash lines). Note that the EBL "upperlimit" model derived in Albert et al. (2008) is based on the same code as presented here, but with a completely different set of parameters, like star-formation rate, dust and gas opacity etc. (see Table 1). Our lower-limit model predicts the smallest correction for extragalactic absorption, as expected, except at very low redshifts $(z<0.2)$, where the Primack (2005) model is slightly above ours. This can be explained by the underestimation in the far-infrared of this model, below the lower limits.

\section{Conclusions}

A lower-limit EBL model was derived utilising the lower limit data from the integration of galaxy number counts from the optical to the far infrared region. The model takes into account timeevolution of galaxies and includes the effect of absorption and re-emission of the interstellar medium. To get such a low EBL, the assumption of a quite low cosmic SFR has to be made, which has a maximum at a redshift of 1.2 of about $0.1 M_{\odot} \mathrm{yr}^{-1} \mathrm{Mpc}^{-3}$ 
T. M. Kneiske and H. Dole: A lower-limit flux for the extragalactic background light
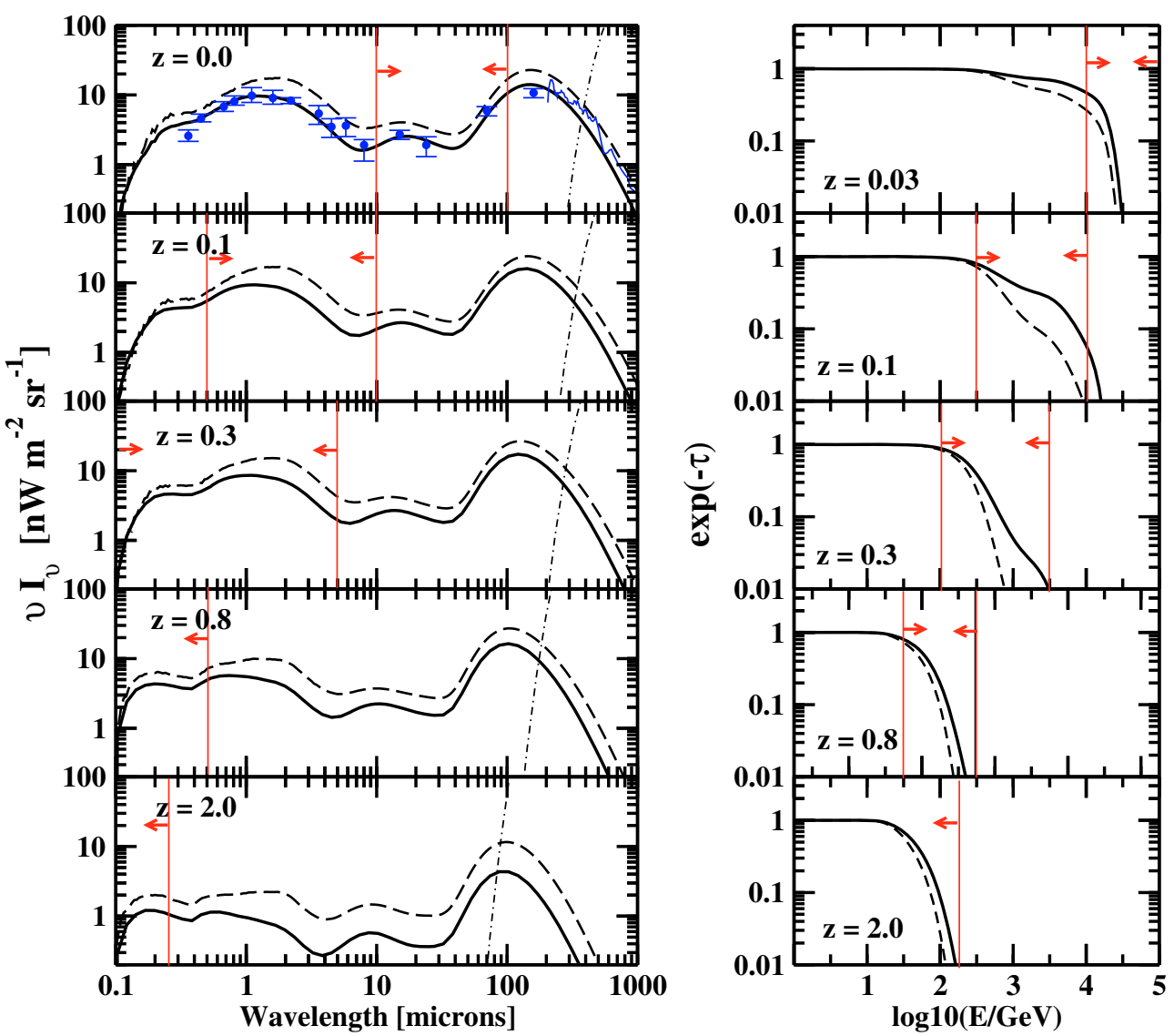

Fig. 4. left: comoving flux of the extragalactic background light at five different redshifts. The solid line represents the lower-limit EBL introduced here, while the dashed line is the old "best-fit" model described in Kneiske et al. (2004). The spectral EBL region responsible for the cut-off at high energy is represented by thin vertical lines and arrows. right: extinction factor of gamma-rays as a function of gamma-ray photon energy at five different redshifts.

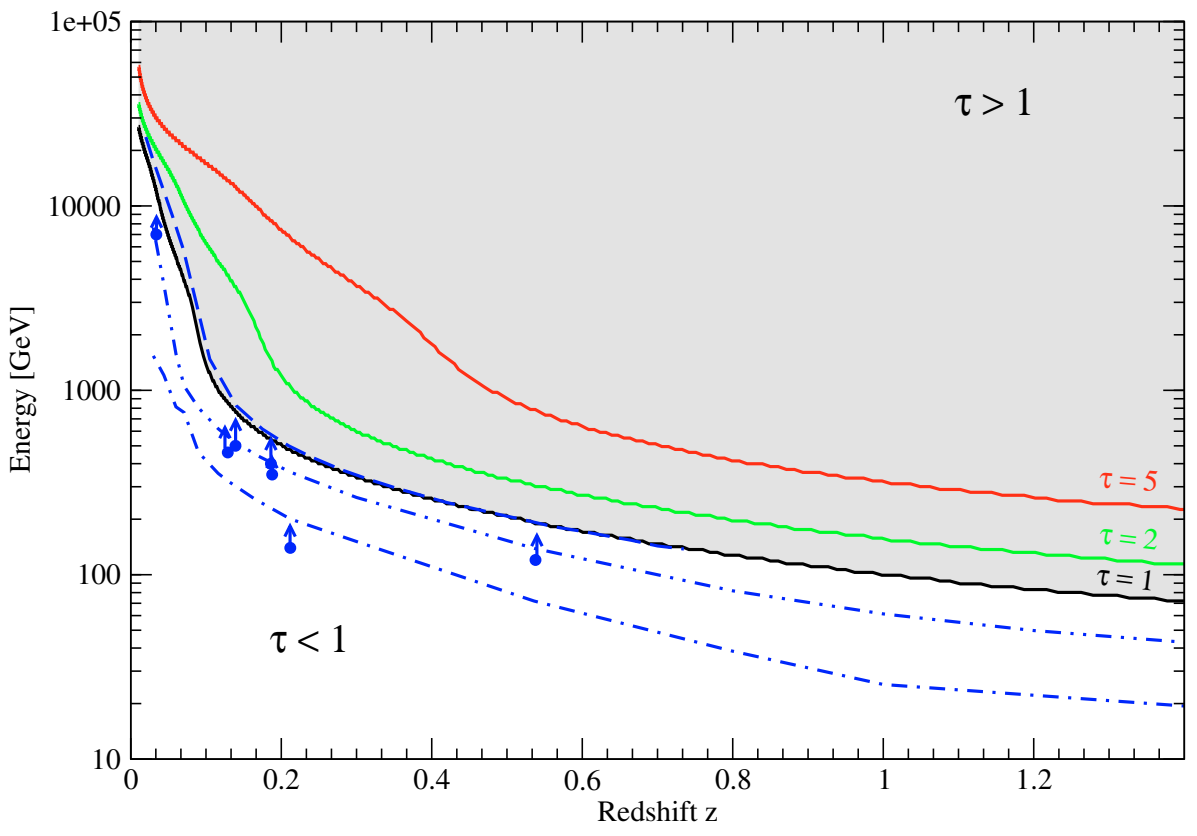

Fig. 5. Gamma-ray horizon $\tau_{\gamma \gamma}\left(E_{c}, z\right)=1$ (black line), $\tau_{\gamma \gamma}\left(E_{c}, z\right)=2$ (green line), $\tau_{\gamma \gamma}\left(E_{c}, z\right)=3$ (red line) for the lower limit EBL model derived in this work. Observed limits (dots) are taken from Fig. 3 of Albert et al. (2008). For comparison, horizons based on three other EBL models are shown in blue, from the bottom: Stecker et al. 2006 (dotdashed), Albert et al. (2008) (dot-dot-dashed) and Primack (2005)(dashed). and falls to a value of about 0.03 at a redshift of 5 . As expected the present-day lower-limit EBL is still below the upper limits derived so far from the process of pair production with very high energy gamma-ray emission by BL Lacs (see red-dashed line in Fig. 3).
This model can be used to calculate the interaction of cosmic-ray particles with ambient photon fields. Cosmic-ray protons loose energy due to pion production with stellar photons if their energy lies in the range between $10^{16}$ and $10^{19} \mathrm{eV}$. Using the EBL model a minimum, guaranteed energy loss of protons can be derived. 


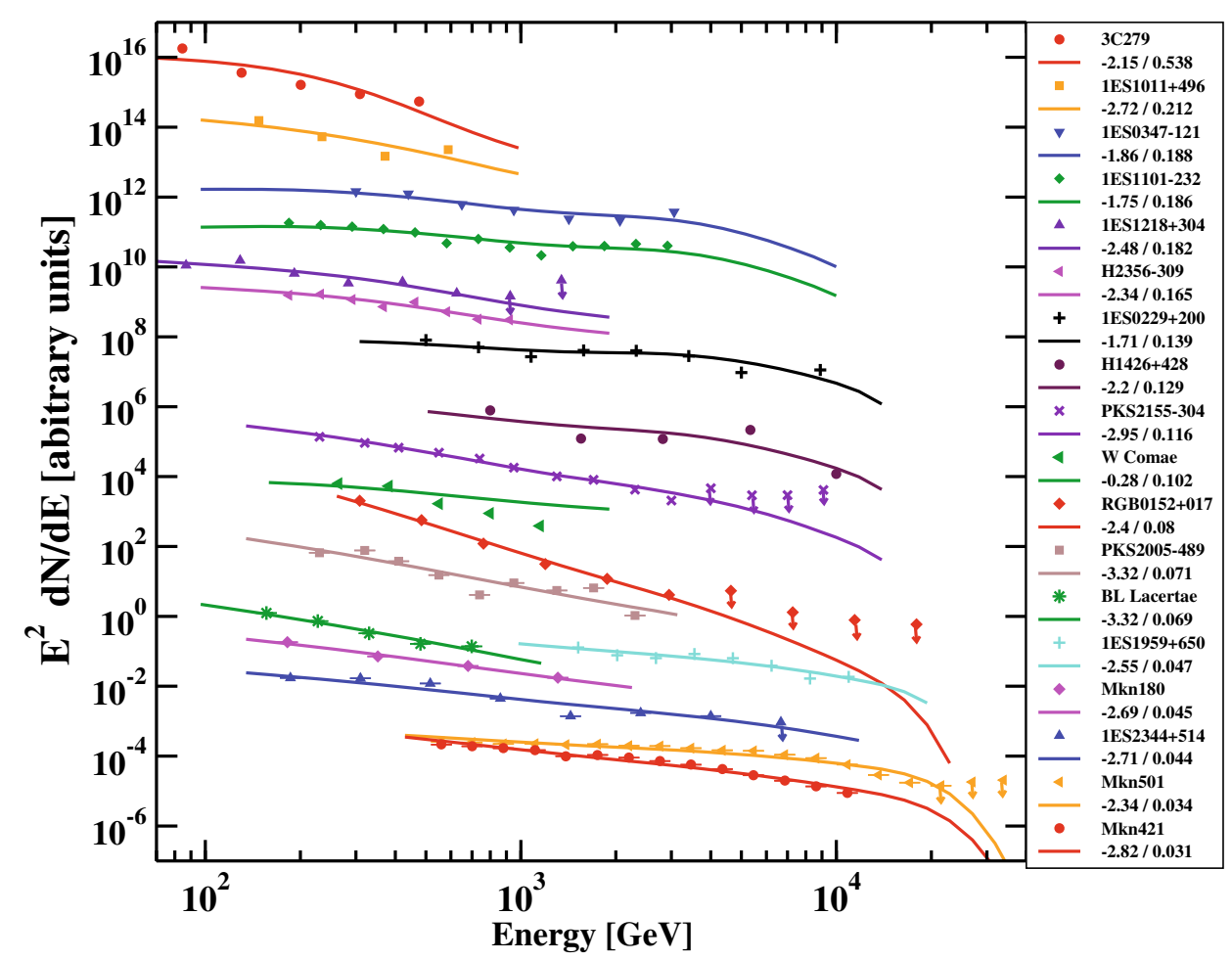

Fig. A.1. Observed spectral energy distributions for blazars (indicated at the right of the figure): dots (data), lines (model). The sources are ordered by their redshift, from high (top) to low redshift (bottom). The total flux is normalized for a better visualization. The lines are model spectra corrected for minimum EBL absorption, described in the text. Numbers on the right indicate the spectral index $\alpha$ and the redshift of the source.

A lower-limit EBL model is also essential to test exotic particle physics scenarios in the universe. Particles like axions (Sanchez-Conde et al. 2009) or hidden photons (Zechlin et al. 2008) can prevent high energy gamma-ray photons from being absorbed. Other mechanisms like Lorentz invariance violations (Protheroe \& Meyer 2000) can only be studied if the uncertainty of the EBL is as small as possible. A minimum absorption due to a guaranteed low energy photon field from galaxies is essential to look for such particles and effects.

This was used to compute the absorption factor for gammarays and observed blazar spectra at some selected redshifts. The Fazio-Stecker relation, which describes the absorption of high energy gamma-rays from extragalactic sources as a function of redshift was also calculated. From this it can be concluded that the lower-limit EBL flux can be used to correct high energy gamma-ray spectra at all redshifts. The minimum correction done with this model seems to lead to realistic intrinsic gammaray spectra of AGN even at high redshift, which can be modeled with standard acceleration scenarios in relativistic jets. Up to now it was only possible to show the agreement between lowerlimit data and indirect upper limits for the present day EBL flux. In this paper we show that also at higher redshift only an EBL close to a lower-limit extragalactic diffuse photon flux, taking into account the complete cosmic evolution of galaxies, agrees with upper limits from high redshift blazar observations.

The recent detection of 3C279 blazar at $z=0.536$ by the MAGIC collaboration Albert et al. (2008), Errando et al. 2009) has brought up the question of the transparency of the Universe to the $\gamma$-rays and of the level of the cosmic infrared background (e.g. Aharonian et al. 2006; Aharonian et al. 2007a,b; Stecker $\&$ Scully 2009). We confirm that the current lower limits of the EBL flux also at a redshift as high as $z=0.536$ are fully compatible with $\gamma$-ray observations, both on the blazar SED and on the $\gamma$-ray horizon.
If in the future EBL limits from $\mathrm{TeV}$ observations become lower, maybe even dropping below the strict lower-limit EBL, the assumptions leading to EBL limits from gamma-ray observations may have to be revised. On the other hand, the discovery of AGN showing a spectral behavior which disagrees with our derived gamma-ray horizon would challenge AGN physics.

The lower limit EBL data, the EBL flux and optical depth as a function of wavelength/energy and redshift are electronically available ${ }^{1}$.

Acknowledgements. We thank Andrew Hopkins for providing us with an electronic form of the CSFR compilation. We thank Wolfgang Rhode and Martin Raue for useful discussions. T.K. acknowledges the support of DFG grant Kn 765/1-2. H.D. acknowledges the support of ANR-06-BLAN-0170.

\section{Appendix A: Application to the SED of blazars}

The lower-limit EBL model is used to calculate spectral energy distribution for observed TeV-blazars. To compare the spectra with the observations, a single power-law is employed with a spectral index indicated below the source name in the table right to Fig. A.1. Figure A.1 shows the spectra of blazars sorted by increasing redshift (from bottom to top) and multiplied by an arbitrary constant to ease visibility. The spectral index and normalization has been taken from a fit of the corrected data points of each source. Then the power law was multiplied by the extinction factor shown in Fig. 4 depending on the redshift of the gamma-ray source. With this method we get a continuous spectrum for each source.

The intrinsic spectra can all be described by power laws with spectral indices still in agreement with very simple jet models in AGN, like the synchrotron-self Compton model (SSC). This was

\footnotetext{
1 In Orsay: http://www.ias.u-psud.fr/irgalaxies/ and in Hamburg: http://www . astroparticle.de.
} 
T. M. Kneiske and H. Dole: A lower-limit flux for the extragalactic background light

not surprising, given the lower limit EBL which has been used. But this might be another indication that the opacity to $\gamma$-rays is still low $(\tau<1)$, even at higher redshift $z \sim 0.5$.

\section{References}

Aharonian, F., Akhperjanian, A. G., Bazer-Bachi, A. R., et al. 2006, Nature, 440, 1018

Aharonian, F., Akhperjanian, A. G., Barres de Almeida, U., et al. 2007a, A\&A, 473, L25

Aharonian, F., Akhperjanian, A. G., Barres de Almeida, U., et al. 2007b, A\&A, 475, L9

Albert, J., Aliu, E., Anderhub, H., et al. 2008, Science, 320, 1752

Balland, C., Devriendt, J., \& Silk, J. 2003, MNRAS, 343, 107

Brown, T. M., Kimble, R. A., Ferguson, et al. 2000, AJ, 120, 1153

Caputi, K. I., Lagache, G., Yan, L., et al. 2007, ApJ, 660, 97

Chary, R., \& Elbaz, D. 2001, ApJ, 556, 562

Chary, R., Casertano, S., Dickinson, M. E., et al. 2004, ApJS, 154, 80

Chelouche, D., Koester, B. P., \& Bowen, D. V. 2007, ApJ, 671, 97

Connolly, A. J., Szalay, A. S., Dickinson, M., et al. 1997, ApJ, 486, L11

Dole, H., Le Floc'h, E., Pérez-Gonzalez, P., et al. 2004, ApJS, 154, 87

Dole, H., Lagache, G., Puget, J.-L., et al. 2006, A\&A, 451, 417

Elbaz, D., Flores, H., Chanial, P., et al. 2002, A\&A, 381, L1

Ellis, R. S., Colless, M., Broadhurst, T., et al. 1996, MNRAS, 280, 235

Errando, M., Bock, R., Kranich, D., et al. 2009, AIPC, 1085, 423

Fazio, G. G., Ashby, M. L. N., Barmby, P., et al. 2004a, ApJS, 154, 39

Fazio, G. G., Hora, J. L., Allen, L. E., et al. 2004b, ApJS, 154, 10

Franceschini, A., Rodighiero, G., Cassata, P., et al. 2006, A\&A, 453, 397

Franceschini, A., Rodighiero, G., \& Vaccari, M. 2008, A\&A, 487, 837

Frayer, D., Fadda, D., Yan, L., et al. 2006, AJ, 131, 250

Frayer, D., Huynh, M. T., Chary, R., et al. 2006, ApJ, 647, L9

Gardner, J. P., Brown, T. M., \& Ferguson, H. C. 2000, ApJ, 542, L79

Goldreich, P., \& Morison, P., JETP, 18, 239

Gould, R. S., \& Schreder, G. 1966, Phys. Ref. Lett., 16, 252

Hauser, M. G., Arendt, R. G., Kelsall, T., et al. 1998, ApJ, 508, 25

Hauser, M. G., \& Dwek, E. 2001, ARA\&A, 39, 249

Hopkins, A. M., \& Beacom J. F. 2006, ApJ, 651, 142
Jelley, J. V. 1966, Phys. Ref. Lett., 16, 479

Kashlinsky, A. 2005, ApJ, 633, 5

King, A. J., \& Rowan-Robinson, M. 2003, MNRAS, 339, 260

Kneiske, T. M., Mannheim, K., \& Hartmann, D. 2002, ApJ, 386, 1

Kneiske, T. M., Bretz, T., Mannheim, K., et al. 2004, ApJ, 413, 807

Lagache, G., Haffner, L. M., Reynolds, R. J., et al. 2000, A\&A, 354, 247

Lagache, G., Dole, H., \& Puget, J.-L. 2003, MNRAS, 338, 555

Lagache, G., Dole, H., Puget, J.-L., et al. 2004, ApJS, 154, 112

Lagache, G., Puget, J.-L., \& Dole, H. 2005, ARA\&A, 43, 727

Levenson, L. R., \& Wright, E. L. 2008, ApJ, 683, 585

Lilly, S. J., Le Fevre, O., Hammer, F., et al. 1996, ApJ, 460, L1

Madau, P., \& Pozzetti, L. 2000, MNRAS, 312, L9

Magdis, G. E., Rigopoulou, D., Huang, J. S., et al. 2008, MNRAS, 386, 11

Malkan, M. A., \& Stecker, F. W. 2001, ApJ, 555, 641

Marleau, F. R., Fadda, D., Storrie-Lombardi, L. J., et al. 2004, ApJS, 154, 66

Mazin, D., \& Raue, M. 2007, A\&A, 471, 439

Nikishov, A. I. 1962, Sov. Phys. JETP, 14, 393

Papovich, C., Dole, H., Egami, E., et al. 2004, ApJS, 154, 70

Pei, Y. C., \& Fall, S. M., \& Hauser, M. G. 1999, ApJ, 522, 604

Pozzetti, L., Madau, P., \& Zamorani, G., et al. 1998, MNRAS, 298, 1133

Primack, J. R., Bullock, J. S., Somerville, R. S., et al. 1999, APh, 11, 93

Primack, J. 2005, AIPC, 745, 23

Primack, J., Gilmore, R. C., \& Somerville, R. S. 2008, AIPC, 1085, 71

Protheroe, R. J., \& Meyer, H. 2000, PhLB, 493, 1

Puget, J.-L., Abergel, A., Bernard, J.-P., et al. 1996, A\&A, 308, L5

Raue, M., Kneiske, T. M., \& Mazin, D. 2009, A\&A, 498, 25

Rodighiero, G., Lari, C., Pozzi, F., et al. 2006, MNRAS, 371, 1891

Salamon, M. H., \& Stecker, F. W. 1998, ApJ, 439, 547

Sánchez-Conde, M. A., Paneque, D., Bloom, E., Prada, F., \& Domínguez, A. 2009, Phys. Rev. D, 79, 123511

Stecker, F. W., Malkan, M. A., \& Scully, S. T., 2006 ApJ, 648, 774

Stecker, F. W., \& Scully, S. T. 2009, ApJ, 691, L91

Thompson, R. I. 2003, ApJ, 596, 748

Thompson, R. I., Eisenstein, D., Fan, X., et al., 2007, ApJ, 657, 669

Totani, T., \& Takeuchi, T. T. 2001, ApJ, 570, 470

Totani, T., Yoshii, Y., Iwamuro, F., et al. 2001, ApJ, 550, L137

Werner, M., Roellig, T. L., Low, F. J., et al. 2004, ApJS, 154, 1

Xu, C. K, Donas, J., Arnouts, S., et al. 2005, ApJ, 619, L11

Zechlin, H.-S., Horns, D., \& Redondo, J. 2008, AIPC, 1085, 727 